\title{
O COMÉRCIO INEXPLORADO ENTRE BRASIL E ASIA: UMA ABORDAGEM ATRAVÉS DO MODELO GRAVITACIONAL ${ }^{\circ}$
}

THE UNEXPLORED TRADE BETWEEN BRAZIL AND ASIA:

AN APPROACH THROUGH THE GRAVITATIONAL MODEL

\author{
Sílvia Letícia Bampi* \\ André Filipe Zago de Azevedo** \\ Magnus dos Reis**
}

enviado: 28 junio 2019 - aceptado: 22 noviembre 2019

\begin{abstract}
Resumo
O presente estudo buscou estimar o comércio potencial do Brasil com o continente asiático no período de 2000 a 2014, utilizando uma amostra de 75 países. Para evitar problemas observados em estudos prévios, estimou-se a equação gravitacional por meio do estimador de Pseudo Máxima Verossimilhança de Poisson (PPML, na sigla inglesa), calculando-se o intervalo de confiança dos coeficientes com o método Delta. O modelo baseou-se em dados em painel com dois conjuntos de efeitos fixos. Em termos absolutos, haveria um potencial de expansão do comércio brasileiro com os países asiáticos selecionados de US\$ 5645 milhões, o equivalente a 7.6\% do comércio médio anual observado no período 2000-2014.

Palavras chave: comércio internacional, modelo gravitacional, Ásia, Brasil. Código JEL: C19, F14, R15.
\end{abstract}

Bampi, S. L., Zago de Azevedo, A. F., \& Reis, M. (2020). O comercio inexplorado entre Brasil e Asia: uma abordagem a traves do modelo gravitacional. Estudios económicos, 37 (75), 51-73.

* Faculdade João Paulo II, Universidade de Passo Fundo (UPF). E-mail: slb.bampi@gmail.com

** Universidade do Vale do Rio dos Sinos (Unisinos).E-mail: aazevedo@unisinos.br, magnusr@unisinos.br 


\begin{abstract}
The present study aimed to estimate Brazil's potential trade with the Asian continent from 2000 to 2014, using a sample of 75 countries. To avoid problems observed in previous studies, the gravitational equation was estimated using the Poisson Pseudo Maximum Likelihood Estimator (PPML), calculating the confidence interval of the coefficients with the Delta method. The model was based on panel data with two sets of fixed effects. In absolute terms, there would be a potential for expansion of Brazilian trade with selected Asian countries of US\$ 5645 million, equivalent to $7.6 \%$ of the average annual trade observed in the period 2000-2014.
\end{abstract}

Keywords: international trade, gravity model, Asia, Brazil. JEL Code: C19, F14, R15. 


\section{INTRODUÇÃO}

A partir do final da década de 1980, o comércio internacional passou a ter um papel mais relevante na economia brasileira, após seu processo de abertura comercial. Intensificou-se nesse período o processo de globalização das economias e a formação de blocos econômicos. Hidalgo e Feistel (2013) destacam que, paralelamente a isto, ocorreu uma mudança na direção dos fluxos comerciais globais, com destaque para o crescimento de economias asiáticas, como China e Índia.

Esse maior intercâmbio comercial dos países asiáticos também foi observado com o Brasil. Ao longo do século XXI, percebe-se um aumento da importância das relações comerciais entre o Brasil e as principais economias asiáticas. Em 2000 , cerca de $11 \%$ das exportações do Brasil e $15 \%$ de suas importações tinham como destino/origem a Ásia, de acordo com dados do MDIC (2015). Em 2014, as relações do Brasil com os países asiáticos já representavam mais de $30 \%$ de suas exportações e importações.

Apesar dos avanços recentes, o Brasil continua sendo uma economia relativamente fechada, quando se considera a participação das exportações e importações no PIB. De acordo com Canuto et al. (2015), o grau de abertura comercial dos parceiros brasileiros do BRICS ${ }^{1}$ atingiu cerca de $50 \%$ do PIB, em 2013, enquanto no Brasil esta proporção foi de apenas $27.6 \%$.

Uma das ferramentas mais utilizadas para estimar o comércio potencial é o modelo gravitacional na sua forma log-linear. Os coeficientes estimados servem para obter o comércio bilateral potencial e compará-lo com o comércio observado. Valores de comércio potencial acima do observado sinalizariam que poderia haver uma expansão do comércio no futuro. No entanto, Egger (2002) questionou esses resultados, alegando que se o modelo fosse corretamente especificado, os valores estimados deveriam convergir aos observados. Breuss e Egger (1999) e Proença et al. (2008) procuraram resolver essa situação ao calcular um intervalo de confiança para testar a hipótese de que a razão entre o comércio potencial e o observado seria maior do que um, usando uma análise em cross-section.

1 O termo BRIC foi difundido pelo economista Jim O' Neill (2001), vinculado ao banco Goldman Sachs. Em seu estudo ele estabeleceu comparações entre estes países e as economias do chamado grupo dos sete (G7) composto por EUA, Canadá, Reino Unido, França, Itália, Alemanha e Japão. Na perspectiva de O'Neill, esse conjunto de países emergentes, que incluía Brasil, Índia, Rússia e China, se tornariam as principais economias do mundo em 2050, ao lado dos Estados Unidos. 
Outra crítica aos estudos anteriores se relaciona ao uso do modelo em forma log-linear, pois conforme destacam Santos Silva e Tenreyro (2011), isso não permite uma estimação consistente dos parâmetros na presença de heterocedasticidade. Os autores sugerem o uso do estimador não-linear de Pseudo Máxima Verossimilhança de Poisson (PPML, na sigla inglesa). Finalmente, Egger (2005) salienta que a estimação desses modelos em cross-section sofre de problemas de especificação, pois não é capaz de lidar com a heterogeneidade bilateral do exportador e do importador, geralmente presente nos fluxos de comércio bilaterais.

Este artigo busca calcular o comércio potencial brasileiro com seus principais parceiros asiáticos incorporando as críticas mencionadas, utilizando o estimador de PPML e calculando um intervalo de confiança para testar a hipótese de que a razão entre o comércio potencial e o observado seria mesmo maior do que um. Além disso, ele se diferencia de Breuss e Egger (1999) e Proença et al. (2008) ao estimar o modelo gravitacional utilizando dados em painel e com dois conjuntos de efeitos fixos, um para os importadores e outro para exportadores em cada ano da amostra, conforme sugerido por Anderson e van Wincoop (2003) para controlar tanto a resistência multilateral como a heterogeneidade bilateral do exportador $\mathrm{e}$ do importador.

O artigo está dividido em cinco seções, iniciando por esta introdução. A seção dois apresenta aspectos relacionados ao modelo gravitacional e ao cálculo do comércio potencial. A terceira seção é reservada para as especificações econométricas, bem como os testes e resultados das estimativas realizadas. Na quarta seção é realizada uma análise sobre a evolução do comércio potencial entre o Brasil e as economias asiáticas. A quinta, por fim, conclui o estudo.

\section{MODELO GRAVITACIONAL E CÁLCULO DO COMÉRCIO POTENCIAL}

Os modelos gravitacionais são procedimentos econométricos utilizados para se avaliar os fluxos de comércio bilaterais. A equação é baseada originalmente nos trabalhos do físico Isaac Newton (1642-1727), que propôs a Teoria da Gravitação Universal. Em sua teoria, Newton definiu que entre dois corpos existe uma força de atração, que é uma relação diretamente proporcional entre o produto da massa dos corpos e inversamente proporcional ao quadrado da distância entre eles.

Baseado nesta equação, Tinbergen (1962) passou a descrever padrões globais de comércio bilateral, seguido por trabalhos de Poyhonen (1963) e Linnemann (1966). Tinbergen (1962) relacionou o volume de comércio ao produto entre o 
tamanho econômico do país e um fator de proporcionalidade, que depende do que ele nomeou como "trade resistance".

O modelo gravitacional possui uma sólida fundamentação teórica, dado que diversos autores derivaram essa equação de diferentes teorias econômicas ${ }^{2}$. Entre os principais, destacam-se os trabalhados de Eaton e Kortum (2002), que derivaram a equação da gravidade pelo lado da oferta por meio de um modelo Ricardiano com bens intermediários, e o de Anderson e van Wincoop (2003), que fundamentaram a equação gravitacional utilizando um modelo de concorrência monopolista.

De acordo com Anderson e van Wincoop (2003), o modelo gravitacional estrutural teórico, derivado pelo lado da demanda, estabelece que, no equilíbrio de longo prazo, o comércio entre dois países deveria ser o seguinte:

$$
m_{i j}=\frac{Y_{i} E_{j}}{Y}\left(\frac{t_{i j}}{\Pi_{i} P_{j}}\right)^{(1-\sigma)}
$$

onde $m_{i j}$ representa as exportações nominais do país $i$ para o destino $j, Y_{i}$ é o valor total da produção do país $i, E_{j}$ é o gasto total no país $j, \sigma$ é o valor total da produção mundial, $Y$ é a elasticidade de substituição entre diferentes variedades, $t_{i j}$ são os custos bilaterais de comércio ${ }^{3}$ entre os países $i$ e $j, \Pi_{i}$ é resistência multilateral externa, que captura o fato de que as exportações do país $j$ para o país $i$ dependem dos custos comerciais em todos os possíveis mercados de exportação, e, finalmente, $P_{j}$ é um índice de preços ao consumidor no país $j$.

Dessa forma, o comércio entre os países $i$ e $j$ é dado pela equação (1) e ele reflete o valor de equilíbrio de longo prazo sugerido pelo modelo gravitacional estrutural teórico. Muito embora esse valor não seja uma variável observável, pode-se utilizar esse modelo na sua forma estocástica para estimar esse comércio de equilíbrio. Ao fazer isso, os eventuais desvios do comércio previsto, estimado por uma equação gravitacional estrutural teórica do comércio observado, refletem potenciais de comércio entre os países.

Especificamente, se o valor previsto for maior que o observado, então haverá potencial de comércio, uma vez que o comércio estimado reflete o comércio que deverá ser alcançando pelos países $i$ e $j$ no longo prazo, dadas as suas características. Portanto,

2 Para maiores informações sobre a fundamental teórica do modelo gravitacional de outras teóricas, veja Yotov et al. (2016).

3 A distância entre os países $i$ e $j$ é utilizada como proxy. 
se o modelo gravitacional utilizado para fazer essas previsões for estrutural, teórico e estiver corretamente especificado, então pode-se considerar que seja o comércio potencial entre os países.

\section{I.1. Comércio Potencial}

O modelo gravitacional tem sido utilizado de forma recorrente para mensurar o comércio potencial bilateral - o comércio esperado entre países a partir de suas características econômicas, geográficas, culturais etc. Egger (2002) define o comércio potencial como sendo a diferença entre o fluxo de comércio observado e 'estimado', o que pode ser interpretado como o potencial de comércio não esgotado. Helmers e Pasteels (2006), por sua vez, caracterizam o potencial de comércio como o resultado das capacidades de exportação e de demanda de importações dos países.

As estimativas do potencial de comércio, como assinalam Helmers e Pasteels (2006), permitem observar o nível de integração dos mercados, sendo que quanto maior a diferença entre o comércio efetivo e o potencial estimado, maior o nível de integração efetiva destes em relação à estimada. Diferenças negativas, ou seja, quando o comércio efetivo é inferior ao previsto, indicam que há potencial de comércio "inexplorado" e que políticas comerciais podem ser adotadas com o objetivo de expandi-lo.

Egger (2002) separa os estudos sobre o cálculo do comércio potencial em duas fases. A primeira, composta pelos trabalhos pioneiros de Wang e Winters (1992) e Hamilton e Winters (1992), ocorreu no âmbito da queda da Cortina de Ferro e da nova integração potencial entre a União Europeia (UE) e os antigos estados membros do $\mathrm{COMECON}^{4}$. Nesses trabalhos, os autores imaginaram que os países do leste europeu, logo após a queda da Cortina de Ferro, ainda se comportavam de forma diferente dos países da Europa ocidental. Assim, eles usaram o modelo gravitacional com os dados do comércio dos países da Europa ocidental para estimar o comércio potencial dos países do leste europeu, a partir dos parâmetros obtidos. Ou seja, na medida que houvesse uma maior integração entre a Europa ocidental e oriental, o comércio entre as regiões deveria aumentar.

4 "Conselho de Assistência Econômica Mútua", fundado em 1949, do qual fazia parte a União Soviética e outros países europeus. 
A segunda fase compreende estudos como os de Baldwin (1994) e Nilsson (2000) que já incluíam os países em transformação na base de dados do modelo gravitacional. Essa abordagem é chamada por Egger (2002) de previsões dentro da amostra. Enquanto o autor considera os trabalhos da primeira fase um esforço valioso de criação de um antimundo para prever os fluxos potenciais de comércio dos países do leste europeu, ele critica a segunda abordagem.

A crítica de Egger (2002) para previsões de comércio potencial dentro da amostra são puramente do ponto de vista econométrico. A primeira delas, ao se referir a projeções dentro da amostra, indica que é muito provável que os modelos gravitacionais em cross-section e estimados por OLS estejam mal especificados, pois ignoram a presença de efeitos fixos dos exportadores e importadores ou não testam sua relevância.

Já na segunda, o autor argumenta que, para um estimador ser consistente e eficiente, é necessário que o resíduo seja ruído branco, ou seja, que ele não apresente qualquer tipo de variação sistemática. Dessa forma, se um estimador revelar grandes diferenças sistemáticas entre os valores observados e previstos na amostra, então, isso deve ser interpretado como uma indicação para erros de especificação e inconsistência dos parâmetros. $\mathrm{E}$ de fato, todos os estimadores utilizados por Egger (2002), FE, RE, Between, HT, RE-AR(1) e HT-AR(1), necessitavam apresentar resíduos normais para serem consistentes e eficientes.

Apesar da veracidade e importância dessas críticas, especialmente no contexto analisado por Egger (2002), algumas considerações devem ser feitas a elas porque este trabalho utiliza não apenas efeitos fixos diferentes, mas também um estimador não utilizado pelo autor.

Em relação à primeira crítica de Egger (2002), deve-se ressaltar que Fally (2015) demonstrou a importância tanto teórica quanto empírica de incluir esses efeitos fixos para estimar um modelo estrutural teórico consistente por meio de PPML, que é o estimador utilizado nesse artigo. Apesar dos argumentos de Fally (2005) sobre a relevância de incluir efeitos fixos, foram realizados testes de significância para os efeitos fixos com objetivo de verificar a validade da crítica de Egger (2002). Especificamente, foram realizados três testes, um para testar a significância dos efeitos fixos anuais dos importadores, outro, a dos efeitos fixos anuais dos exportadores e o último para a significância conjunta deles.

Não obstante, ao optar pela PPML, obtêm-se estimativas consistentes dos parâmetros, mesmo com erros heterocedásticos, além de possibilitar a inclusão dos 
fluxos de comércio com valores iguais a zero, conforme salientaram Santos Silva e Tenreyro (2006). Além de ser consistente na presença de efeitos fixos, conforme Cameron e Trivedi (2013), mesmo que a variância condicional não seja proporcional à média condicional, ou seja, nos casos de sub ou superdispersão dos dados, a PPML ainda será consistente 5 .

Já a segunda crítica de Egger (2002) é mitigada porque, para que a PPML seja um estimador consistente, basta que a média condicional, $\mathrm{E}\left[y_{i} \mid x\right]=\exp \left(x_{i} \beta\right)$, esteja corretamente especificada, conforme salientaram Santos Silva e Tenreyro (2006, p. 645). Para verificar essa questão, os autores sugerem utilizar o Teste Reset, que foi utilizado nesse artigo. Portanto, nenhuma hipótese sobre os resíduos é necessária para que as estimativas dos parâmetros populacionais sejam consistentes. Para alcançar eficiência, basta que a variância condicional esteja corretamente especificada ${ }^{6}$.

\section{ESPECIFICAÇÕES ECONOMÉTRICAS E RESULTADOS}

O fluxo bilateral de comércio assume papel de variável dependente e será explicado pelas variáveis independentes. Para representar o fluxo bilateral de comércio, neste estudo, utilizaram-se dados de importação bilateral $\left(m_{i j t}\right)$, seguindo exemplo de Hamilton e Winters (1992), justificando-se no fato das importações serem um fluxo controlado com maior rigor devido à necessidade de saída de divisas. Os dados das importações bilaterais em dólares correntes foram obtidos da base de dados do UN COMTRADE (2016). A especificação das variáveis, suas origens e o sinal esperado na estimativa são apresentados no Quadro 1.

As variáveis Distância $(\mathrm{Km})$, Extensão Territorial $\left(\mathrm{Km}^{2}\right)$ e as dummies para Fronteira Comum, Costa Litoral, Idioma Comum, Colonização Comum foram extraídas das bases do Centre d'Études Prospectives et d'Informations Internationales (CEPII, 2016), enquanto as variáveis relacionadas à participação em APCs 7 foram extraídas de OMC (2016).

5 Ademais, a PPML é consistente mesmo que a variável dependente não seja distribuída de acordo com a distribuição de Poisson.

6 Alternativamente, Breuss e Egger (1999) e Proença et al. (2008) calcularam um intervalo de confiança para testar a hipótese de que a razão entre o comércio potencial e o observado seria maior do que um, usando uma análise em cross-section.

7 Foram considerados os principais APCs, incluindo acordos bilaterais dos países asiáticos considerados na amostra, com base em OMC (2016): Common Market for Eastern and South Africa (COMESA), Southern African Development Community (SADC), European Union (EU), North American Free Trade Agreement (NAFTA), Southern Common Market (MERCOSUR), ASEAN 
Quadro 1. Variáveis consideradas

\begin{tabular}{|c|c|c|c|c|}
\hline Série & Variável & Unidade & Sinal Esperado & Origem \\
\hline $\begin{array}{l}\text { Importações } \\
\text { Bilaterais } \\
\text { (Variável } \\
\text { dependente) }\end{array}$ & & U\$\$ & & $\begin{array}{c}\text { UN } \\
\text { COMTRADE } \\
(2016)\end{array}$ \\
\hline $\begin{array}{l}\text { Distância entre } \\
\text { as capitais dos } \\
\text { países }\end{array}$ & & Quilômetros & Negativo & CEPII (2016) \\
\hline $\begin{array}{l}\text { Extensão } \\
\text { Territorial }\end{array}$ & & Quilômetros ${ }^{2}$ & Negativo & CEPII (2016) \\
\hline $\begin{array}{l}\text { Fronteira } \\
\text { Terrestre }\end{array}$ & & Dummy & Positivo & CEPII (2016) \\
\hline Costa litoral & & Dummy & Positivo & CEPII (2016) \\
\hline Idioma Comum & & Dummy & Positivo & CEPII (2016) \\
\hline $\begin{array}{l}\text { Colonização } \\
\text { Comum }\end{array}$ & & Dummy & Positivo & CEPII (2016) \\
\hline $\begin{array}{l}\text { Participação } \\
\text { em APCs }\end{array}$ & & Dummy & $\begin{array}{l}\text { Ambíguo (Mas } \\
\text { geralmente } \\
\text { positivo) }\end{array}$ & OMC (2016) \\
\hline
\end{tabular}

Fonte: elaboração própria.

Com o objetivo de estimar o comércio potencial do Brasil com o continente asiático, foram considerados os fluxos de comércio em ambos os sentidos (Brasil como importador e exportador), portanto entre o Brasil e as principais economias da região, conforme destacado no Quadro 2. Para a obtenção dos resultados da análise, os fluxos de comércio são estimados um a um e, posteriormente, agregados, para

Free Trade Area (AFTA), The Australia New Zealand Closer Economic Agreement (ANZCERTA), Asia Pacific Trade Agreement (APTA), Central American Common Market (CACM), Andean Community (CAN), Common Economic Zone (CEZ), Commonwealth of Independent States (CIS), European Free Trade Association (EFTA), Gulf Cooperation Council (GCC), Latin American Integration Association (LAIA), Pan-Arab Free Trade Area (PAFTA), South Asian Free Trade Agreement (SAFTA) e Acordos Bilaterais entre Coreia-Singapura, Coreia-Peru, Coreia-EUA, Coreia-Turquia, China-Nova Zelândia, China-Singapura, China-Peru e China-Suíça. 
análise do fluxo comercial total brasileiro com as principais economias do sudeste asiático e da Ásia como um todo.

Quadro 2. Fluxos Bilaterais de Comércios Analisados

\begin{tabular}{cc}
\hline Brasil X China & China X Brasil \\
Brasil X Filipinas & Filipinas X Brasil \\
Brasil X Indonésia & Indonésia X Brasil \\
Brasil X Malásia & Malásia X Brasil \\
Brasil X Tailândia & Tailândia X Brasil \\
Brasil X Coréia do Sul & Coréia do Sul X Brasil \\
Brasil X Índia & Índia X Brasil \\
Brasil X Japão & Japão X Brasil \\
\hline Brasil X Singapura & Singapura X Brasil \\
Brasil X Vietnã & Vietnã X Brasil \\
\hline
\end{tabular}

Fonte: elaboração própria.

As estimações do modelo gravitacional consideraram uma base de dados formada por 75 países $^{8}$, em um período de 15 anos (2000-2014), séries com frequência anuais, resultando em um conjunto de 83250 observações. A seleção dos países levou em conta as 75 principais economias, em termos de comércio acumulado durante o período da amostra, que reportaram dados bilaterais de comércio. Essas economias em 2014 representaram 94\% do comércio mundial (UN COMTRADE, 2016).

A respeito das importações bilaterais, foram identificados 2120 fluxos zerados de comércio, o que representou $2.55 \%$ do total da amostra. Assim, para a transformação logarítmica, necessária à estimação por meio de MQO, a amostra deveria

8 África do Sul, Alemanha, Arábia Saudita, Argélia, Argentina, Austrália, Áustria, Bahrein, Bielorrússia, Bélgica, Bolívia, Brasil, Bulgária, Camboja, Canadá, Cazaquistão, Chile, China, Colômbia, Coreia do Sul, Croácia, Dinamarca, Equador, Egito, El Salvador, Estados Unidos da América, Emirados Árabes Unidos, Estônia, Eslováquia, Eslovênia, Espanha, Etiópia, Finlândia, França, Filipinas, Grécia, Guatemala, Hungria, Índia, Indonésia, Israel, Itália, Japão, Letônia, Líbano, Lituânia, Luxemburgo, Malásia, México, Marrocos, Moçambique, Nova Zelândia, Noruega, Omã, Holanda, Paraguai, Peru, Polônia, Portugal, Reino Unido, República Tcheca, România, Rússia, Singapura, Sri Lanka, Suécia, Suíça, Tailândia, Tanzânia, Turquia, Ucrânia, Uruguai, Vietnã, Venezuela, Zâmbia. 
ser reduzida para um total de 79 142, o que acabaria por desconsiderar importantes informações em relação a baixos níveis de comércio que podem estar relacionados, por exemplo, a fatores econômicos, geográficos ou culturais. Da mesma forma, poderiam ser atribuídos valores baixos para os dados zerados, permitindo a transformação logarítmica (Linnemann, 1966; Wang \& Winters, 1992), contudo, valores positivos pequenos tornam-se números negativos grandes quando em log, acabando por resultar em estimações viesadas e inconsistentes.

Santos Silva e Tenreyro (2006) destacam que se o modelo for log-linearizado ou se for feita transformação/exclusão na variável dependente devido aos zeros existentes na amostra, o modelo pode incorrer em viés nas estimativas. No entanto, Wooldridge (2010) salienta que, dada a utilização da variável dependente em nível, se sua configuração for estritamente positiva, é provável que ela possua distribuição condicional heterocedástica ou concentrada, e a transformação logarítmica poderia aliviar ou até mesmo eliminar esses problemas. Outra solução seria a utilização do estimador de Poisson e Pseudo-Máxima Verossimilhança (PPML), que permite a utilização dos fluxos bilaterais de comércio iguais a zero, permitindo executar o formato não-linear do modelo utilizando a variável dependente em nível, controlando a heterocedasticidade9. Já a heterogeneidade bilateral do importador e exportador passar a ser controlada por meio da utilização de dados em painel com a inclusão dos efeitos fixos.

No tópico seguinte são apresentadas as definições do modelo e os testes aos quais ele foi submetido para seu ajuste e melhor especificação.

\section{II.1. Definições do Modelo e Testes de Especificação}

Wei (1996), Deardorff (1998) e Anderson e van Wincoop (2003) criticaram o modelo gravitacional tradicional, estimado através de dados agrupados (pooled) ou cross-section, por apresentarem problemas de especificação ao ignorar a "resistência multilateral" e o "isolamento" dos países. Egger (2005) acrescentou que a estimação desses modelos através de dados agrupados ou cross-section sofre de problemas de especificação, uma vez que ela não é capaz de lidar com a heterogeneidade bilateral do exportador e do importador, que é provável que esteja presente nos fluxos de comércio bilaterais. Segundo Egger (2005), ignorar tais características pode produzir estimativas viesadas. Visando corrigir esses problemas de

9 Outra opção seria seguir a abordagem sugerida por Helpman et al. (2008). 
má especificação, Mátyás (1997), Anderson e van Wincoop (2003), entre outros, sugerem a estimação da equação gravitacional em dados em painel e efeitos fixos.

Apesar da existência de certo consenso na literatura sobre a necessidade de estimar o modelo gravitacional em dados em painel para controlar a heterogeneidade, Cheng e Wall (1999) salientaram que os pesquisadores divergem sobre qual seria a melhor forma de especificar o efeito fixo. Os autores sugerem a inclusão de dois efeitos fixos, um para cada par de países $\left(\alpha_{i j}\right)$ e o outro é específico de cada ano $t\left(\alpha_{t}\right)$. Essa abordagem usualmente utiliza o PIB e a População dos importadores e exportadores como variáveis independentes no modelo, incorrendo no que Baldwin e Taglioni (2006) denominaram de Gold Medal Mistake, uma vez que são ignorados os termos que capturam a resistência multilateral, propostos por Anderson e van Wincoop (2003). Em vez de utilizar o PIB e a População, a literatura sugere duas alternativas para mitigar o risco de obter um viés nas estimações: $i)$ criar índices de isolamento para os exportadores e importadores, propostos por Anderson e van Wincoop (2003) ou ii) criar efeitos fixos anuais para importadores e exportadores.

Nesse sentido, uma forma alternativa de especificar os efeitos fixos foi proposta por Baldwin e Taglioni (2006) e Magee (2008). Nessa especificação três efeitos fixos são incluídos na estimativa, sendo: um constante no tempo para cada par de países $\left(\alpha_{\mathrm{ij}}\right)$, outro efeito fixo para o exportador $j$ em cada período de tempo $t$ $\left(\alpha_{\mathrm{j} t}\right)$ e um terceiro efeito fixo específico para o importador $i$ em cada tempo $\left.t\left(\alpha_{\mathrm{it}}\right)\right)^{10}$

Entretanto, a abordagem com três efeitos fixos proposta por Baldwin e Taglioni (2006) traz algumas restrições, dentre elas a impossibilidade de estimar o impacto das principais variáveis usualmente utilizadas nos modelos gravitacionais, tais como a distância. Por esse motivo, optou-se pela utilização de dois efeitos fixos $\left(\alpha_{\mathrm{jt}}\right.$ e $\left.\alpha_{\mathrm{it}}\right)$. Os efeitos fixos para os importadores (i) e exportadores $(j)$ em cada ano $t$ controlam a resistência multilateral e o isolamento dos países.

A regressão foi realizada por meio de dados em painel com dois efeitos fixos e por meio de dois estimadores: i) Pseudo Máxima Verossimilhança de Poisson (PPML), como proposto por Santos Silva e Tenreyro (2006), não-linear, e ii) $\mathrm{MQO}^{11}$, linear. A equação estimada pela PPML é representada pela Equação (2), enquanto a definida para o estimador de MQO é a (3), como segue:

10 Baier e Bergstrand (2007) argumentaram que a inclusão do efeito fixo para cada par de países controla a endogeneidade da política comercial.

11 Antes de tomar o logaritmo da variável dependente, foi somado o valor de um a todos os valores da variável dependente. 
$m_{i j t}=\exp \left[\alpha_{0}+\alpha_{i t}+\alpha_{j t}+\beta_{1} \log\right.$ Dist $_{i j}+\beta_{2} \log$ Area $_{i}+$ $\beta_{3} \log$ Area $_{j}+\beta_{4}$ Idioma $_{i j}+\beta_{5}$ Adj $_{i j}+\beta_{6} L_{i}+\beta_{7} L_{j}+\beta_{8}$ Col $_{i j}+$ $\left.\sum_{k=9}^{33} \beta_{k} A P C_{k i j t}\right] \times \varepsilon_{i j t}$

$\log m_{i j t}=\alpha_{0}+\alpha_{i t}+\alpha_{j t}+\beta_{1} \log$ Dist $_{i j}+\beta_{2} \log$ Area $_{i}+$ $\beta_{3} \log$ Area $_{j}+\beta_{4}$ Idioma $_{i j}+\beta_{5}$ Adj $_{i j}+\beta_{6} L_{i}+\beta_{7} L_{j}+\beta_{8}$ Col $_{i j}+$ $+\sum_{k=9}^{33} \beta_{k} A P C_{k i j t}+\log \varepsilon_{i j t}$

onde $m_{i j t}$ é o escalar das importações do país $i$ procedentes do exportador $j$ no tempo $t ; \alpha_{0}$ é a constante, $\alpha_{i t}$ é o escalar do efeito fixo do importador $i$ no tempo $t ; \alpha_{j t}$ é o escalar do efeito fixo do exportador $j$ no tempo $t$; $D i s t_{i j}$ é o escalar da distância entre o exportador $j$ e o importador $i ;$ Area $_{i}$ e $A r e a_{j}$ são os escalares, respectivamente, das áreas territoriais do importador $i$ e exportador $j$; Idioma $a_{i j}$ é a dummy que assume valor 1 se o exportador $j$ e o importador $i$ têm um idioma em comum; $A d j_{i j}$ é a dummy que assume valor 1 se o exportador $j$ e o importador $i$ possuem uma fronteira; $L_{i}$ e $L_{j}$ são as dummies que assumem valor 1 se o importador $i$ e o exportador $j$, respectivamente, não têm litoral; $\operatorname{Col}_{i j}$ é a dummy que assume valor 1 se o exportador $j$ e o importador $i$ tiveram uma relação colonial; $A P C_{k i j t}$ são as dummies dos APCs; $\alpha_{0}, \alpha_{i t}, \alpha_{j t}, \beta_{1}, \beta_{2}, \ldots, \beta_{33}$ são parâmetros a serem estimados e $\varepsilon_{i j t}$ é o escalar do erro idiossincrático.

Antes de analisar as estimativas, os resultados dos testes descritos anteriormente, sob o modelo estimado pela PPML, serão apresentados. Conforme observado na Tabela 1, o p-valor apresentado não rejeita a hipótese nula, indicando que o modelo está corretamente especificado ${ }^{12}$. Dessa forma, as eventuais diferenças entre o comércio vigente e o previsto não se devem à má especificação do modelo, mas sim ao comércio potencial existente entre os parceiros bilaterais. No entanto, as conclusões sobre o potencial comercial devem levar em consideração a variação amostral da razão entre o comércio projetado e o observado, considerando os intervalos de confiança ou o teste de hipótese de que esse índice é maior que um.

\section{II.2. Resultados das Estimativas}

Os resultados das estimativas, utilizando ambos os estimadores (PPML e MQO), podem ser encontrados na Tabela 1. Uma vez que o estimador PPML é

12 A hipótese nula do Teste Reset afirma que o modelo está corretamente especificado. 
mais robusto para estimar a equação gravitacional, conforme demonstram Santos Silva e Tenreyro (2006) e Santos Silva e Tenreyro (2015), a análise dos resultados é feita tendo como referência o modelo baseado nesse estimador. Os coeficientes estimados no modelo foram utilizados, na sequência, para obtenção dos resultados do cálculo do comércio potencial entre os pares de países selecionados.

Os coeficientes para área territorial via PPML foram positivos, opondo-se ao esperado, ou seja que quanto maior a área, tende-se a reduzir o comércio internacional, dada a maior abundância de recursos no país, como afirma Azevedo (2004). $\mathrm{Na}$ estimativa via MQO a área do exportador teve sinal esperado, no entanto, o coeficiente não apresentou significância. Da mesma forma, as variáveis idioma comum e adjacência tendem a aumentar as relações de comércio ${ }^{13}$, considerando as estimativas através de ambos os estimadores. A adjacência não foi significativa por meio de MQO.

A presença de costa litoral no exportador, nos resultados para ambos os estimadores, reduz o fluxo de comércio internacional, contrapondo-se ao esperado. A presença de litoral no importador funciona como um facilitador de comércio e, portanto, aumenta esse fluxo, de acordo com Piermartini e Teh (2005) e Plummer et al. (2010), no entanto esse coeficiente não foi significativo. O coeficiente da distância também teve o sinal esperado, ou seja, quanto maior a distância entre os países, menor será o comércio entre eles.

Os resultados para as dummies de participação em APCs, no caso em que o exportador e o importador integram o acordo, sinalizam que a maior parte deles tem ampliado os fluxos de comércio. Utilizando o estimados PPML, apenas as dummies de participação no ASEAN, CAN e PAFTA não apresentaram significância, além das dummies dos acordos bilaterais entre Coréia e EUA, Coréia e Turquia; China e Suíça. No caso do estimador de MQO não foram significativas as dummies de participação no Nafta, UE, SAFTA, ANZCERTA e as dummies dos acordos bilaterais entre Coréia e Singapura, Coréia e EUA, Coréia e Turquia; China e Suíça e China e Nova Zelândia.

13 Esse resultado condiz com o encontrado em Breuss e Egger (1999). 
O COMÉRCIO INEXPLORADO ENTRE BRASIL E ASIA: UMA ABORDAGEM ATRAVÉS DO MODELO...

Tabela 1. Resultados das Estimativas do Modelo

\begin{tabular}{|c|c|c|c|c|}
\hline \multirow{3}{*}{$\begin{array}{l}\text { Variável } \\
\text { Distância }\end{array}$} & \multicolumn{2}{|l|}{ PPML } & \multicolumn{2}{|l|}{ MQO } \\
\hline & \multirow{2}{*}{$\frac{\text { Coef. }}{-0.574^{* * *}}$} & Erro-Padrão & \multicolumn{2}{|c|}{ Coef. Erro-Padrão } \\
\hline & & $(0.033)$ & $-0.999 * * *$ & $(0.087)$ \\
\hline Área Importador & $0.422 * * *$ & $(0.136)$ & $2.018 * * *$ & $(0.151)$ \\
\hline Área Exportador & 0.082 & $(0.063)$ & -0.195 & $(0.142)$ \\
\hline Idioma Comum & $0.234 * * *$ & $(0.067)$ & $1.194 * * *$ & $(0.139)$ \\
\hline Adjacência & $0.402 * * *$ & $(0.062)$ & 0.229 & $(0.206)$ \\
\hline Importador sem Litoral & 0.219 & $(0.185)$ & -0.266 & $(0.242)$ \\
\hline Exportador sem Litoral & $-2.472 * * *$ & $(0.573)$ & $-7.398 * * *$ & $(0.491)$ \\
\hline Colonização Comum & 0.037 & $(0.151)$ & $1.522 * * *$ & $(0.392)$ \\
\hline Asean & 0.157 & $(0.150)$ & $0.552 *$ & $(0.335)$ \\
\hline Mercosul & $1.249 * * *$ & $(0.173)$ & $0.872 *$ & $(0.519)$ \\
\hline Nafta & $0.869 * * *$ & $(0.152)$ & 0.355 & $(0.631)$ \\
\hline $\mathrm{EU}$ & $0.682 * * *$ & $(0.087)$ & -0.007 & $(0.067)$ \\
\hline Sadac & $1.872 * * *$ & $(0.409)$ & $4.581 * * *$ & $(0.742)$ \\
\hline Efta & $-1.148 * *$ & $(0.502)$ & $-1.139 * * *$ & $(0.167)$ \\
\hline $\mathrm{GCC}$ & $-0.979 * *$ & $(0.415)$ & $-0.565 * *$ & $(0.226)$ \\
\hline CEZ & $2.020 * * *$ & $(0.173)$ & $-0.404 * *$ & $(0.179)$ \\
\hline Safta & $2.171 * * *$ & $(0.529)$ & -0.173 & $(0.144)$ \\
\hline CIS & $-0.164 * * *$ & $(0.048)$ & $-0.311 *$ & $(0.167)$ \\
\hline $\mathrm{Cacm}$ & $2.406^{* * *}$ & $(0.366)$ & $4.738 * * *$ & $(0.551)$ \\
\hline Apta & $-0.863 * * *$ & $(0.241)$ & $-1.025 * *$ & $(0.408)$ \\
\hline Laia & $0.265^{*}$ & $(0.144)$ & $1.155^{* * *}$ & $(0.262)$ \\
\hline Anzcerta & $1.161 * * *$ & $(0.190)$ & 0.217 & $(0.277)$ \\
\hline Can & $0.766^{* * *}$ & $(0.245)$ & $1.528 * * *$ & $(0.440)$ \\
\hline Comesa & 0.764 & $(0.609)$ & $2.163 * * *$ & $(0.663)$ \\
\hline Pafta & 0.261 & $(0.281)$ & $2.015 * * *$ & $(0.383)$ \\
\hline Coreia_Singapura & $0.509 * * *$ & $(0.150)$ & 0.197 & $(0.143)$ \\
\hline Coreia_Peru & $0.700 * * *$ & $(0.181)$ & $0.481 * * *$ & $(0.114)$ \\
\hline Coreia_EUA & 0.103 & $(0.169)$ & 0.007 & $(0.178)$ \\
\hline Coreia_Turquia & -0.276 & $(0.346)$ & 0.029 & $(0.110)$ \\
\hline
\end{tabular}




\begin{tabular}{lllll} 
China_Nova Zelândia & $0.273^{*}$ & $(0.146)$ & 0.131 & $(0.210)$ \\
\hline China_Singapura & $-0.480^{* * *}$ & $(0.162)$ & $-0.496^{* * *}$ & $(0.174)$ \\
\hline China_Peru & $0.785^{* * *}$ & $(0.165)$ & $0.303^{*}$ & $(0.157)$ \\
\hline China_Suíça & 0.169 & $(0.441)$ & 0.559 & $(0.357)$ \\
\hline Constante & $18.112^{* * *}$ & $(2.215)$ & Dropped & \\
\hline Efeito Fixo Anual em $i$ e $j$ & Sim & & Sim & \\
\hline Número de Obs. & 83,248 & 83,250 & \\
\hline $\mathrm{R}^{2}$ & 0,917 & 0,794 & \\
\hline Teste de signif. para $\alpha_{i t}$ & {$[0.0000]$} & - & \\
\hline Teste de signif. para $\alpha_{j t}$ & {$[0.0000]$} & - & \\
\hline Teste de signif. para $\alpha_{i t}$ e $\alpha_{j t}$ & {$[0.0000]$} & - & \\
Teste Reset & {$[0.2544]$} & - & \\
\hline
\end{tabular}

Fonte: elaboração própria.

Notas: *,** e *** correspondem aos níveis de significância de $90 \%, 95 \%$ e 99\%, respectivamente. Erros padrões robustos clusterizados entre parênteses e p-valor dos testes de especificação em colchetes.

Depois de analisadas as estimativas resultantes do modelo gravitacional, na sequência serão apresentados os resultados do cálculo de comércio potencial entre o Brasil e os parceiros asiáticos selecionados, objetivo principal deste estudo, baseando-se nas propostas de Breuss e Egger (1999) e Proença et al. (2008).

\section{COMÉRCIO POTENCIAL ENTRE BRASIL E ÁSIA}

Até o presente momento, foram apresentadas as especificações econométricas necessárias e as estimativas dos coeficientes para o cálculo de comércio potencial. Nesta seção, apresentam-se as estimativas do comércio potencial entre o Brasil e algumas das principais economias asiáticas entre 2000 e 2014. Para tanto, os coeficientes obtidos no modelo foram combinados com os dados reais de cada par de países, obtendo-se assim o fluxo bilateral potencial de cada par. Foram considerados os fluxos médios por parceiro, em todo o período abrangido pela amostra, para analisar os fluxos bilaterais potenciais e o comércio efetivo entre os pares de países. Esses resultados podem ser encontrados na Tabela 2.

Em 2014, houve um expressivo aumento do comércio efetivo entre o Brasil e as economias asiáticas. As importações brasileiras da Ásia (considerando os 
países selecionados) cresceram mais de 764\%, passando de U\$\$ 7748 milhões em 2000 para U\$\$ 66993 milhões, enquanto as importações asiáticas do Brasil tiveram uma elevação de $1030 \%$. Para o mesmo ano, as dez economias asiáticas selecionadas representaram $27.02 \%$ das importações mundiais e $28.33 \%$ das exportações (UN COMTRADE, 2016).

Seguindo a proposta de Breuss e Egger (1999) e Proença et al. (2008), a análise do comércio potencial do país $i$ com o país $j$ deve ser feita considerando a razão entre o comércio estimado $\left(\widehat{m}_{i j t}\right)$ e o comércio observado $\left(m_{i j t}\right)$. Se essa razão for maior que um, então significa que o país $j$ não está exportando para o país $i$ o valor do comércio que foi estimado pelo modelo estrutural teórico. Por outro lado, uma razão menor que um significa que não existe comércio potencial a ser explorado entre os países $i$ e $j$. Portanto, deve-se interpretar a existência de potencial de comércio entre dois países sempre quando a razão entre o comércio previsto e o observado for maior que um porque isso reflete um desvio do equilíbrio de longo prazo, de modo que o comércio deverá convergir para seu nível de equilíbrio, ou seja, para aquele estimado pelo modelo gravitacional.

No entanto, para obter inferências mais robustas, deve-se levar em consideração a variância amostral do comércio estimado, ou seja, é necessário levar em conta o intervalo de confiança dessas estimativas. Dessa forma, foram construídos os intervalos de confiança de $95 \%$ para cada estimativa de comércio utilizando o método Delta, conforme sugestão de Cameron e Trivedi (2013) e que já foi utilizado por Proença et al. (2008) no contexto de comércio potencial.

A partir disso, foram obtidos, para cada par de países ij no tempo $t$, os limites inferior e superior dos intervalos de confiança desses comércios estimados para que, posteriormente, fossem calculadas as médias desses limites para cada par de nações, além de calcular a média do comércio observado no período. Por fim, para cada par de países foi calculada a razão do limite inferior do comércio médio estimado sobre o valor do comércio médio observado ${ }^{14}$.

Nos fluxos bilaterais examinados, levando em conta a média das importações com cada parceiro nos 15 anos da amostra (Tabela 2), os valores estimados para as importações brasileiras ficaram abaixo das importações efetivas quando os parceiros foram China (11.7\%), Coréia do Sul (31.1\%) e Índia (36.5\%). Já as importações estimadas dos parceiros asiáticos do Brasil ficaram acima das efetivas

14 Mesma ideia de cálculo foi utilizada para encontrar a razão do limite superior. 
em oito dos dez fluxos considerados, com exceção de China e Coréia do Sul. Desta forma, parece haver espaço para uma ampliação tanto das importações brasileiras dos países asiáticos, bem como significativa oportunidade de expansão das exportações brasileiras para esses destinos.

Tabela 2. Potencial de Comércio entre o Brasil e as economias asiáticas selecionadas considerando a média dos fluxos entre 2000 a 2014 (em milhões de US\$)

\begin{tabular}{|c|c|c|c|c|c|c|c|c|}
\hline \multirow[b]{2}{*}{ Parceiro Asiático } & \multicolumn{8}{|c|}{ Brasil como Importador } \\
\hline & Estimado & Efetivo & Diferença & Diferença \% & $\begin{array}{c}\text { Limite } \\
\text { Superior }\end{array}$ & $\begin{array}{c}\text { Limite } \\
\text { Inferior }\end{array}$ & $\begin{array}{c}\text { Razão_Limite } \\
\text { Superior }\end{array}$ & $\begin{array}{c}\text { Razão_Limite } \\
\text { Inferior }\end{array}$ \\
\hline China & 14.079 & 15.953 & -1.874 & $-11,7 \%$ & 18.100 & 11.000 & 1,1342 & 0,6876 \\
\hline Japão & 6.656 & 4.893 & 1.763 & $36,0 \%$ & 8.210 & 5.400 & 1,6789 & 1,1052 \\
\hline Coréia do Sul & 3.281 & 4.760 & -1.479 & $-31,1 \%$ & 4.450 & 2.420 & 0,9320 & 0,5075 \\
\hline Índia & 1.753 & 2.762 & -1.009 & $-36,5 \%$ & 2.250 & 1.370 & 0,8158 & 0,4957 \\
\hline Malásia & 1.650 & 1.199 & 451 & $37,6 \%$ & 2.160 & 1.260 & 1,7970 & 1,0460 \\
\hline Indonésia & 1.534 & 939 & 595 & $63,4 \%$ & 2.070 & 1.140 & 2,1978 & 1,2026 \\
\hline Singapura & 1.367 & 784 & 583 & $74,4 \%$ & 1.780 & 1.050 & 2,2621 & 1,3354 \\
\hline Tailândia & 1.331 & 998 & 333 & $33,4 \%$ & 1.870 & 1.210 & 1,5949 & 1,0297 \\
\hline Vietnã & 590 & 360 & 230 & $63,9 \%$ & 918 & 380 & 2,5432 & 1,0517 \\
\hline Filipinas & 514 & 281 & 233 & $82,9 \%$ & 695 & 381 & 2,4822 & 1,3621 \\
\hline \multirow[b]{2}{*}{ Parceiro Asiático } & \multicolumn{8}{|c|}{ Brasil como Exportador } \\
\hline & Estimado & Efetivo & Diferença & Diferença \% & $\begin{array}{c}\text { Limite } \\
\text { Superior }\end{array}$ & $\begin{array}{c}\text { Limite } \\
\text { Inferior }\end{array}$ & $\begin{array}{c}\text { Razão_Limite } \\
\text { Superior } \\
\end{array}$ & $\begin{array}{c}\text { Razão_Limite } \\
\text { Inferior }\end{array}$ \\
\hline China & 11.442 & 24.560 & -13.118 & $-53,4 \%$ & 16.400 & 8.020 & 0,6654 & 0,3254 \\
\hline Japão & 6.771 & 6.753 & 18 & $0,3 \%$ & 9.910 & 4.650 & 1,4714 & 0,6906 \\
\hline Coréia do Sul & 3.179 & 3.383 & -204 & $-6,0 \%$ & 4.880 & 2.080 & 1,4387 & 0,6136 \\
\hline Índia & 2.782 & 2.011 & 771 & $38,3 \%$ & 4.160 & 1.860 & 2,0625 & 0,9230 \\
\hline Singapura & 2.240 & 1.052 & 1.188 & $112,9 \%$ & 3.250 & 1.550 & 3,0689 & 1,4629 \\
\hline Tailândia & 1.558 & 1.295 & 263 & $20,3 \%$ & 2.470 & 986 & 1,9033 & 0,7609 \\
\hline Malásia & 1.262 & 909 & 353 & $38,8 \%$ & 1.890 & 843 & 2,0726 & 0,9229 \\
\hline Indonésia & 1.231 & 1.068 & 163 & $15,3 \%$ & 1.820 & 834 & 1,7137 & 0,7846 \\
\hline Vietnã & 571 & 461 & 110 & $23,9 \%$ & 894 & 365 & 1,9222 & 0,7854 \\
\hline Filipinas & 488 & 220 & 268 & $121,8 \%$ & 766 & 311 & 3,4878 & 1,4171 \\
\hline
\end{tabular}

Fonte: elaboração própria.

Para as exportações brasileiras com destino a Singapura, evidenciou-se um potencial de expansão de $112.9 \%$ (1180 milhões). A expansão do comércio com o país beneficiaria vários elos da cadeia produtiva brasileira, do agronegócio à indústria, pois os produtos manufaturados possuem a maior participação nas exportações brasileiras para aquele país, além de semimanufaturados e produtos básicos, como carnes. Somando-se os valores de ambos os países, há um potencial de ampliação das exportações brasileiras de US\$ 1456 milhões. 
Pelo lado das importações brasileiras, haveria um comércio ainda a ser explorado para boa parte dos países asiáticos examinados, incluindo Indonésia, Japão, Malásia, Filipinas, Singapura, Tailândia e Vietnã. Assim como ocorreu com as exportações, o maior potencial de aumento das importações brasileiras ocorreria com Filipinas e Singapura, chegando a $83.3 \%$ e $74.3 \%$, respectivamente. Em termos absolutos, o maior volume de crescimento das importações brasileiras ocorreria com o Japão, chegando a US\$ 1763 milhões. Considerando a soma de comércio inexplorado pelo lado das importações brasileiras, ela chegaria US\$ 4189 milhões.

Portanto, em termos absolutos, haveria um potencial de expansão do comércio brasileiro com os países asiáticos selecionados de US\$ 5645 milhões, o equivalente a 7.6\% do comércio médio anual observado no período 2000-2014. Assim, mesmo que o mercado asiático tenha elevado sua participação no intercâmbio comercial com a economia brasileira ao longo dos anos, há ainda potencial de expansão de comércio do Brasil com a Ásia. Para pesquisas futuras, seria importante entender a origem da diferença entre os fluxos estimados e observados. Essa diferença pode estar relacionada a políticas específicas implementadas ou a mudanças de políticas dos países envolvidos.

\section{CONCLUSÕES}

O presente estudo objetivou estimar o comércio potencial do continente asiático com o Brasil no período de 2000 a 2014, calculando os comércios potenciais dos fluxos selecionados. Para evitar problemas observados em estudos prévios, estimou-se a equação gravitacional estrutural teórica, por meio de dados em painel com dois efeitos fixos, por meio do estimador de Pseudo Máxima Verossimilhança de Poisson (PPML). Seguindo a proposta de Breuss e Egger (1999) e Proença et al. (2008), considerou-se que havia comércio inexplorado apenas quando a razão entre o comércio estimado e o comércio observado do país $i$ com o país $j$ foi maior que um, porque isso reflete um desvio do equilíbrio de longo prazo, de modo que o comércio deverá convergir para seu nível de equilíbrio, ou seja, para aquele estimado pelo modelo gravitacional.

Buscando obter inferências mais robustas, levou-se em consideração a variância amostral do comércio estimado, ou seja, o intervalo de confiança dessas estimativas. Dessa forma, foram construídos os intervalos de confiança de $95 \%$ para cada estimativa de comércio, utilizando o método Delta, conforme sugestão de Proença et al. (2008). 
Considerando-se as exportações brasileiras, haveria potencial de crescimento apenas para Filipinas e Singapura, totalizando US\$ 1456 milhões. Em relação às importações brasileiras, o potencial de ganho se amplia, tanto em quantidade de parceiros como no volume total. Nesse caso, haveria um comércio ainda a ser explorado com sete dos dez países asiáticos examinados e o montante chegaria a US\$ 4189 milhões.

Assim, apesar do recente aumento do intercâmbio comercial com países daquela região, a partir de 2000, há ainda potencial de expansão de comércio do Brasil com a Ásia. Em termos absolutos, haveria um potencial de aumento do comércio brasileiro com os países asiáticos selecionados de US\$ 5645 milhões, o equivalente a 7.6\% do comércio médio anual observado no período 2000-2014.

\section{REFERÊNCIAS BIBLIOGRÁFICAS}

Anderson, J. E. (1979). A Theoretical Foundation for the Gravity Equation. The American Economic Review, 69 (1), 106-116.

Anderson, J. E., \& van Wincoop, E. (2003). Gravity with gravitas: a solution to the border puzzle. The American Economic Review, 93 (1), 170-192.

Azevedo, A. F. Z. (2004). Mercosur: Ambitious Policies, Poor Practices. Brazilian Journal of Political Economy, 24 (4), 584-601.

Baier, S. L., \& Bergstrand, J. H. (2007). Do free trade agreements actually increase members' international trade? Journal of International Economics, 71 (1), $72-95$.

Balassa, B. (1978). Exports and economic growth. Journal of Development Economy, 5 (2), 181-189.

Baldwin, R. E. (1994). Potential trade Patterns. Toward an integrated Europe. London: Central Economic Policy Research, 69-101.

Baldwin, R., \& Taglioni, D. (2006). Gravity for dummies and dummies for gravity equations. National Bureau of Economic Research, Working Paper No. 12516. Disponível em https://www.nber.org/papers/w12516.

Bergstrand, J. H. (1985). The Gravity Equation in International Trade: Some Microeconomic Foundations and Empirical Evidence. The Review of Economics and Statistics, 67 (3), 474-481.

Breuss, F., \& Egger P. (1999). How Reliable are Estimations of East-West Trade Potential Based on Cross-Section Gravity Analyses? Empirica, 26 (2), 81-94.

Cameron, A. C., \& Trivedi, P. K. (2013). Regression Analysis of Count Data. Cambridge, UK: Cambridge University Press. 
Canuto, O., Fleischhaker, C., \& Schellekens, P. (2015). O curioso caso da falta de abertura do Brasil ao comércio. RBCE, 122, 20-25.

CEPII (2016). Centre d'Études Prospectives et d'Informations Internationales Database. Disponível em http://www.cepii.fr/cepii/en/bdd_modele/bdd.asp. Chaney, T. (2008). Distorted gravity: the intensive and extensive margins of international trade. The American Economic Review, 98 (4), 1707-1721.

Cheng, I.-H., \& Wall, H. J. (1999). Controlling for heterogeneity in gravity models of trade and integration. Federal Reserve Bank of St. Louis, Working Paper No. 1999-010E.

Deardorff, A. (1998). Determinants of bilateral trade: Does gravity work in a neoclassical world? En Frankel, J. A. The Regionalization of the World Economy (pp. 7-32). Chicago: University of Chicago Press.

Eaton, J., \& Kortum, S. (2002) Technology, Geography and Trade. Econometrica, 70 (5), 1741-1779.

Egger, P. (2002). An Econometric View on the Estimation of Gravity Model and the Calculation of Trade Potential. The World Economy, 25 (2), 297-312.

Egger, P. (2005). Alternative techniques for estimation of cross-section gravity models. Review of International Economics, 13 (5), 881-891.

Fally, T. (2015). Structural Gravity and Fixed Effects. Journal of International Economics, 97 (1), 76-85.

Fundo Monetário Internacional (FMI) (2015). World Economic Outlook Databases (WEO). Disponível em http://www.imf.org/external/index.htm.

Greene, W. H. (2013). Export Potential for U.S. Advanced Technology Goods to India Using a Gravity Model Approach. Working Paper No. 2013-03B. Washington, D. C.: Office of Economics, International Trade Commission.

Hamilton, C., \& Winters, L. A. (1992). Opening up international trade with Eastern Europe. Economic Policy, 7 (14), 77-116.

Helmers, C., \& Pasteels, J. (2006). Assessing Bilateral Trade Potential at the Commodity Level: An Operational Approach. ITC, Working Paper Nov. 2006. Geneva, Switzerland: Market Analysis Section.

Helpman, E., Melitz, M., \& Rubinstein, Y. (2008). Estimating trade flows: trading partners and trading volumes. The Quarterly Journal of Economics, 123(2), 441-487.

Hidalgo, A., \& Feistel, P. (2013). Mudanças na Estrutura do Comércio Exterior Brasileiro: Uma Análise sob a Ótica da Teoria de Heckscher-Ohlin. Estudos Econômicos (São Paulo), 43 (1), 79-108.

Linnemann, H. (1966). An econometric study of international trade flows. Amsterdam: North-Holland Publishing Company.

Magee, C. S. P. (2008). New measures of trade creation and trade diversion. Journal of International Economics, 75 (2), 349-362. 
Mátyás, L. (1997). Proper Econometric Specification of the Gravity Model. The World Economy, 20, 363-368.

Ministério Do Desenvolvimento, Indústria e Comércio Exterior (MDIC) (2015). Estatísticas de Comércio Exterior. En Documentos, datos y recursos. Disponível em http://www.mdic.gov.br/sitio/.

Nilsson, L. (2000). Trade integration and the EU economic membership criteria. European Journal of Political Economy, 16 (4), 807-27.

Piermartini, R., \& Teh, R. (2005). Demystifying modelling methods for trade policy. Discussion Paper No. 10. Geneva, Switzerland: World Trade Organization.

Plummer, M., Cheong, D., \& Hamanaka, S. (2010). Methodology for Impact Assessment of Free Trade Agreements. Manila: Asian Development Bank. Pöyhönen, P. (1963). A Tentative Model for the Volume of Trade between Countries. Weltwirtschaftliches Archiv. 90, 93-99.

Proença, I., Fontoura, M., \& Martínez-Galán, E. (2008). Trade in the enlarged European Union: a new approach on trade potential. Portuguese Economic Journal, 7 (3), 205-224.

Santos Silva, J. M. C., \& Tenreyro, S. (2006). The log of gravity. Review of Economics and Statistics, 88 (4), 641-658.

Santos Silva, J. M. C., \& Tenreyro, S. (2011). Further simulation evidence on the performance of the Poisson pseudo-maximum likelihood estimator. Economics Letters, 112 (2), 220-222.

Santos Silva, J. M. C., \& Tenreyro, S. (2015). Trading partners and trading volumes: implementing the helpman-melitz-rubinstein model empirically. Oxford Bulletin of Economics and Statistics, 77 (1), 93-105.

Tinbergen, J. (1962). Shaping the World Economy: Suggestions for an International Economic Policy Books. Nueva York: Twentieth Century Fund.

United Nation Commodity Trade Statistics Database (UN COMTRADE) (2016). Base de datos COMTRADE de la ONU. Disponível em https://comtrade. un.org/data/.

United Nations Conference on Trade and Development (UNCTAD) (2015). World Investment Report 2015: Reforming International Investment Governance. New York: United Nations.

Wang, Z. K., \& Winters, L. A. (1992). The trading potential of Eastern Europe. Journal of Economic Integration, 7 (2), 113-136.

Wei, S.-J. (1996). Intra-national versus international trade: How stubborn are nations in global integration? Working Paper No. 5531. Cambridge, Massachusetts: National Bureau of Economic Research.

Wooldridge, J. M. (2010). Econometric analysis of cross section and panel data. Cambridge, Massachusetts: MIT Press. 
World Bank (2015). World Bank Open Data. En Documentos, datos y recursos. Disponível em http://www.worldbank.org/.

World Economic Forum (WEF) (2016). Global Economic Outlook. En Documentos, datos y recursos. Disponível em https://www.weforum.org/.

World Trade Organization (2016). International trade statistics. En Documentos, datos y recursos. Disponível em https://www.wto.org/.

Yotov, Y. V., Piermartini, R., Monteiro, J.-A., \& Larch, M. (2016). An Advanced Guide to Trade Policy Analysis: The Structural Gravity Model. Geneva: World Trade Organization Publications.

(C) 2020 por los autores; licencia no exclusiva otorgada a la revista Estudios económicos. Este artículo es de acceso abierto y distribuido bajo los términos y condiciones de una licencia Atribución-No Comercial 4.0 Internacional (CC BY-NC 4.0) de Creative Commons. Para ver una copia de esta licencia, visite http://creativecommons.org/licenses/by-nc/4.0 
